REVISTA PROYECCIONES $\mathrm{N}^{\circ} 10: 157-159$

Diciembre 1985 - ISSN 0716-0917

Jornada Matemáticas, Agosto 1985.

\title{
RESULTADOS GENERALES DE APROXIMACION EN LOS ESPACIOS DE SOBOLEV
}

PEDRO HUERTA MARIN*

Si $(K, P, \Sigma)$ es un elemento finito de Lagrange, a toda función de Finida sobre $\mathrm{K}$ se asocia la función $\Pi \mathrm{T}, \mathrm{P}$ - interpolando de Lagrange de $\mathrm{v}$ sobre $\Sigma$. Por estudiar el error de interpolación v - Iv.

Sea $\mathrm{K}$ un subconjunto compacto de $\mathbf{R}^{11}$, cunexo, $\stackrel{\circ}{\mathrm{K}} \neq \phi:$ : Denotaremos por $H^{m}(K)$ al espacio de Sobolev $H^{m}(\dot{K})$. Si E es un subespacio de $H^{m}(K)$, el espacio cuociente $H^{m}(K) / E$ es el conjunto de las clases de equivalencia $\dot{V}$ de las funciones de $\mathrm{H}^{\mathrm{m}}(\mathrm{K})$ módulo 1 a relación de equivalencia $\mathbb{R}$, $\left(v_{1} \mathbb{R} v_{2} \Longleftrightarrow v_{1}-v_{2} G E\right)$.

Un primer resultado importante es: si E es un subespacio cerra do de $H^{\mathrm{m}}(\mathrm{K})$, el espacio $\mathrm{H}^{\mathrm{m}}(\mathrm{K}) / \mathrm{E}$ dotado de la norma cuociente

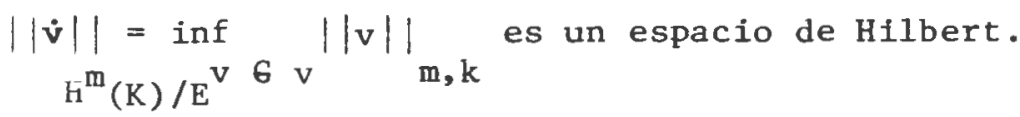

* Dedartamento de Matemáticas, Universidad de Antofagasta, Antofagasta. 
Se puede introducir entonces el espacio $\mathrm{H}^{\mathrm{m}}(\mathrm{K}) / \mathrm{P}_{\mathrm{k}}\left(\mathrm{k} \geqslant 0, \mathrm{P}_{\mathrm{k}}\right.$ : es pacio de los polinomios de $\mathbb{R}^{\mathbf{n}}$ en $\mathbb{R}$ de grado $\leqslant k$ ), nos interesa dotar a este espacio de una norma más manejable que la norma cuociente. Para este efecto se prueba:

TEOREMA: Sea $\mathrm{K}$ un subconjunto compacto y conexo de $\mathbf{R}^{\mathrm{n}}$ de frontera $\xi^{1}$ por pedazos. Entonces, para todo entero $k \geqslant 0$, la aplicación $\dot{\mathrm{v}} \rightarrow|\dot{\mathrm{v}}|_{\mathrm{k}+1, \mathrm{k}}=|\mathrm{v}|_{\mathrm{k}+1, \mathrm{k}}$, donde $\mathrm{v}$ es un representante cual quiera de $\dot{\mathrm{v}}$ en $\mathrm{H}^{\mathrm{k}+1}(\mathrm{~K})$ es una norma sobre $\mathrm{H}^{\mathrm{k}+1}(\mathrm{~K}) / \mathrm{P}_{\mathrm{k}}$ equivalen te a la norma cuociente.

Esto permite entregar el siguiente resultado general de aproxima ción en los espacios de Sobolev.

TEOREMA: Sea K un subconjunto compacto y conexo de $\mathbb{R}^{\mathrm{n}}$ de frontera $\xi^{1}$ por pedazos y sea II un operador lineal continuo de $\mathrm{H}^{k+1}(\mathrm{~K})$ sobre $\mathrm{H}^{\mathrm{m}}(\mathrm{K}), \quad 0 \leqslant \mathrm{~m} \leqslant \mathrm{k}+1$, tal que: $\quad \forall \mathrm{p} \in \mathrm{P}_{\mathrm{k}}, \Gamma_{\mathrm{p}}=\mathrm{p}$.

Entonces existe una constante $C=C(K, \Pi)$ tal que:

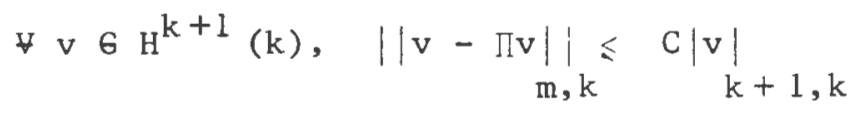

Con el propósito de explicitar la dependencia de la constante c en función de las características geométricas de $\mathrm{K}$, se introduce un subconjunto compacto $\hat{\mathrm{K}}$ de $\mathbb{R}^{\mathrm{n}}$, conexo y de frontera $\ell^{l}$ por pedazos, que actuará como dominio de referencia. Se supone además que existe una transformación afin invertible $F$, tal que $x=F(\hat{x})=\hat{B x}+b$ ( $B$ es una matriz invertible de orden $n$ y $b$ es un vector de $\mathbb{R}^{n}$ ) y además $K=F(\hat{K})$.

\section{Denotando por:}

$\mathrm{h}_{\mathrm{k}}$ : Mäximo de las distancias euclidianas entre 2 puntos de $\mathrm{K}$ (respectivamente $\hat{\mathrm{h}}_{\mathrm{k}}$ ) 
$\rho_{k}$ : Diámetro máximo de las esferas contenidas en $\mathrm{K}$ (respectivamente $\rho_{k}$ )

y usando 1a norma espectral:

$$
|| \mathbf{B}||=\sup _{\substack{\xi \\ \xi} \mathbb{R}^{\mathrm{n}}} \frac{|\mathrm{B} \xi|}{|\xi|},\left(|\xi|: \text { norma euclidiana de } \xi \text { en } \mathbf{R}^{\mathrm{n}}\right)
$$

se pueden probar las desigualdades:
i) ||$B||<-\frac{1}{\hat{\rho}_{k}} h_{k} \quad y$
ii) $\left\|B^{-1}\right\| \leqslant \hat{h}_{k} \frac{1}{\rho_{k}}$, mediante

las cuales se puede justificar el resultado principal.

TEOREMA: Sea $\hat{\mathrm{K}}$ un subconjunto compacto y conexo de $\mathbb{R}^{\mathrm{n}}$ de frontera $\xi^{1}$ por pedazos y sea $\hat{T}$ un operador lineal continuo de $H^{k+1}(\hat{K})$ en $H^{m}(\hat{K})$, $0 \leqslant \mathrm{~m} \leqslant \mathrm{k}+1$, tal que: $\forall \hat{\mathrm{p}} \in \mathrm{P}_{\mathrm{k}}, \hat{\Pi} \hat{\mathrm{p}}=\hat{\mathrm{p}}$.

Si $K$ es un subconjunto de $\mathbb{R}^{\mathrm{n}}$ tal que existe una transformación afín invertible $F$ de $\mathbb{R}^{n}$ en $\mathbb{R}^{n}$ para la cual $K=F(\hat{K})$ y si el operador II está definido por: $\forall \vee \in H^{k+1}(K), \hat{I} \hat{v}=\hat{I} \hat{v}$.

Entonces existe una constante $C$ independiente de $F$ tal que $\forall \mathrm{v} \in \mathrm{H}^{\mathrm{k}+1}(\mathrm{~K}),\left.\left|\mathrm{v}-\prod_{\mathrm{m}, \mathrm{k}} \leqslant \mathrm{C} \frac{\mathrm{h}_{\mathrm{k}}^{\mathrm{k}+1}}{\rho_{\mathrm{k}}^{\mathrm{m}}}\right| \mathrm{v}\right|_{\mathrm{k}+1, \mathrm{~K}}$. 